\title{
Vaccines are essential in campaigns against HIV and TB, say researchers
}

There is an error in the sixth paragraph of this News story ( $B M J$ 2012;344:e3625, doi:10.1136/bmj.e3625) by Peter Moszynski. The last line should have read: "Despite recent efforts, incidence [not "prevalence"] of the disease was falling by only $1 \%$ a year, and even this progress was threatened by the emergence of drug resistance and coinfection with HIV."

Cite this as: BMJ 2012;344:e3742

๑ BMJ Publishing Group Ltd 2012 